\title{
Validation of an Offshore Wind Atlas Using the Satellite Data Available at the Coastal Regions of Portugal
}

\author{
R. Marujo', P. Costa', M. Fernandes', T. Simões' and A. Estanqueiro' \\ 'National Laboratory of Energy and Geology, Estrada do Paço do Lumiar, 22 I649-038 Lisboa \\ (Portugal), raquel.marujo@Ineg.pt, paulo.costa@Ineg.pt, miguel.fernandes@Ineg.pt, \\ teresa.simoes@Ineg.pt, ana.estanqueiro@Ineg.pt
}

Received May 10, 20I3;Accepted May 26, 2013

\begin{abstract}
In this study a validation methodology for regional mesoscale model simulations when ingested with surface wind data inferred from satellite sources around Continental Portugal is evaluated. Observational wind data from a "quasi" offshore anemometric mast located in the Berlenga Island - near Peniche region - was used for the validation study. Satellite sources of wind data under assessment are the ones being used in the EC funded FP7 NORSEWInD project, such as the QuikSCAT and SAR. The validation study evolves 10 years of full wind data, starting in January 2000 to December 2009. The evaluation was performed in two different spatial validation approaches. Results from this study indicate that the wind satellite data has good quality to be assimilated on high resolution mesoscale model simulations particularly the ones concerned with long term behavior of the wind field near the coastal areas.
\end{abstract}

\section{INTRODUCTION}

Mesoscale model simulations are a very promising tool to characterize the wind flow and for the production of wind atlases for wind power studies. The output of these models consists on a group of several meteorological variables for different height levels on a grid that covers the area under investigation. Generally, the results provided by those models contain systematic errors that are not exclusively dependent on the physical parameterizations but are in fact influenced by the topography shape, the spatial resolution of the simulated grid, interpolation errors between observational and grid model points, among others. To interpret and characterize these errors a spatial statistical methodology, using the observational data as reference, was applied and evaluated.

The purpose of this work is to validate the wind field from a long term simulation provided by the $W R F[1]$ mesoscale model, using spatial observational surface wind data from the QuikSCAT [2] satellite data for Portugal. The observational wind data from the satellite was given as input to two different spatial interpolation methodologies used for validation and evaluation of the quality of the simulated results, namely, the known Kriging interpolation method and a newer Composite interpolation method [3]. To evaluate the validation quality 